\title{
The biological in vitro effect and selectivity of aromatic dicationic compounds on Trypanosoma cruzi
}

\author{
Cristiane França da Silva', Patrícia Bernadino da Silva', Marcos Meuser Batista', \\ Anissa Daliry ${ }^{1}$, Richard R Tidwell ${ }^{2}$, Maria de Nazaré Correia Soeiro'1/+
}

${ }^{1}$ Laboratório de Biologia Celular, Instituto Oswaldo Cruz-Fiocruz, Av. Brasil 4365, 21045-900 Rio de Janeiro, RJ, Brasil

${ }^{2}$ Department of Pathology and Laboratory Medicine, University of North Carolina at Chapel Hill, Chapel Hill, NC, USA

Trypanosoma cruzi is a parasite that causes Chagas disease, which affects millions of individuals in endemic areas of Latin America. One hundred years after the discovery of Chagas disease, it is still considered a neglected illness because the available drugs are unsatisfactory. Aromatic compounds represent an important class of DNA minor groove-binding ligands that exhibit potent antimicrobial activity. This study focused on the in vitro activity of 10 aromatic dicationic compounds against bloodstream trypomastigotes and intracellular forms of T. cruzi. Our data demonstrated that these compounds display trypanocidal effects against both forms of the parasite and that seven out of the 10 compounds presented higher anti-parasitic activity against intracellular parasites compared with the bloodstream forms. Additional assays to determine the potential toxicity to mammalian cells showed that the majority of the dicationic compounds did not considerably decrease cellular viability. Fluorescent microscopy analysis demonstrated that although all compounds were localised to a greater extent within the kinetoplast than the nucleus, no correlation could be found between compound activity and kDNA accumulation. The present results stimulate further investigations of this class of compounds for the rational design of new chemotherapeutic agents for Chagas disease.

Key words: aromatic compounds - Trypanosoma cruzi - chemotherapy - Chagas disease

Chagas disease is a neglected tropical illness caused by the protozoan Trypanosoma cruzi. Although Carlos Chagas described it 100 years ago (1909), it is still an important public health problem in Latin America (Rocha et al. 2007). The main clinical symptoms of Chagas disease are cardiac and/or digestive alterations and the overall prevalence of the disease is about 12-14 million cases, which makes it the major cause of cardiac infectious disease in endemic areas (Stewart et al. 2005, Dias 2007). In addition, despite fruitful attempts to control vectorial and blood transmission, Chagas disease still lacks prophylactic therapies and effective chemotherapeutic schemes (Rodrigues Coura \& De Castro 2002, Dias 2007). Nifurtimox and benznidazole are used for the treatment of Chagas disease (Urbina 2002); although they are effective for the treatment of acute infections, they present moderate activity, exhibit undesirable side effects and require long dosing schedules for chronic infections, which frequently necessitate the cessation of treatment (Jannin \& Villa 2007, Soeiro et al. 2009). In addition, the pharmaceutical industries have given little attention to the design and development of new antiparasitic compounds aromatic dicationic compounds represent a class of DNA minor-groove binding ligands

Financial support: FAPERJ, Pensa Rio/FAPERJ, CNPq, PAPES V/ Fiocruz, CPPD

+ Corresponding author: soeiro@ioc.fiocruz.br

Received 6 August 2009

Accepted 13 April 2010 that exhibit high activity against a variety of pathogens, such as bacteria, fungi and protozoa (Werbovetz 2006, Wilson et al. 2008). Recent data showed that diamidines and related compounds, such as the reversed amidines, present considerable efficacy against $T$. cruzi both in vitro (De Souza et al. 2004, Silva et al. 2007a) and in vivo (De Souza et al. 2006a, da Silva et al. 2008) and induce striking alterations on the parasite mitochondrion-kinetoplast complex (De Souza et al. 2006b, Silva et al. 2007b). In this context, the present study investigated the activity of 10 newly synthesised aromatic dicationic compounds on trypomastigotes and intracellular amastigotes, the clinically relevant forms of $T$. cruzi and the toxicity of these compounds in cardiac cells. Due to the intrinsic fluorescent characteristics of these compounds, we also studied their sub cellular distributions to evaluate their preferred targets in T. cruzi.

\section{MATERIALS AND METHODS}

Compounds - The dicationic aromatic compounds 1MAA119 (Compound 1), 25DAP013 (Compound 2), 14SMB013 (Compound 3), 10SAB092 (Compound 4), 10SAB031 (Compound 5), 11SAB081 (Compound 6), 12SMB032 (Compound 7), 150OXD049 (Compound 8), 18SMB092 (Compound 9) and 18SMB096 (Compound 10) (Fig. 1) were synthesised in the laboratory of R.R.T. and the previously reported protocol (Daliry et al. 2009) was used to assess the effectiveness of aromatic compounds with different shapes, cationic centres and effective motifs. Stock solutions of the drugs $(5 \mathrm{mM})$ were freshly prepared in dimethyl sulfoxide and the final solvent concentration in the assays never exceeded $0.6 \%$, which is not toxic for either parasites or mammalian cells. 


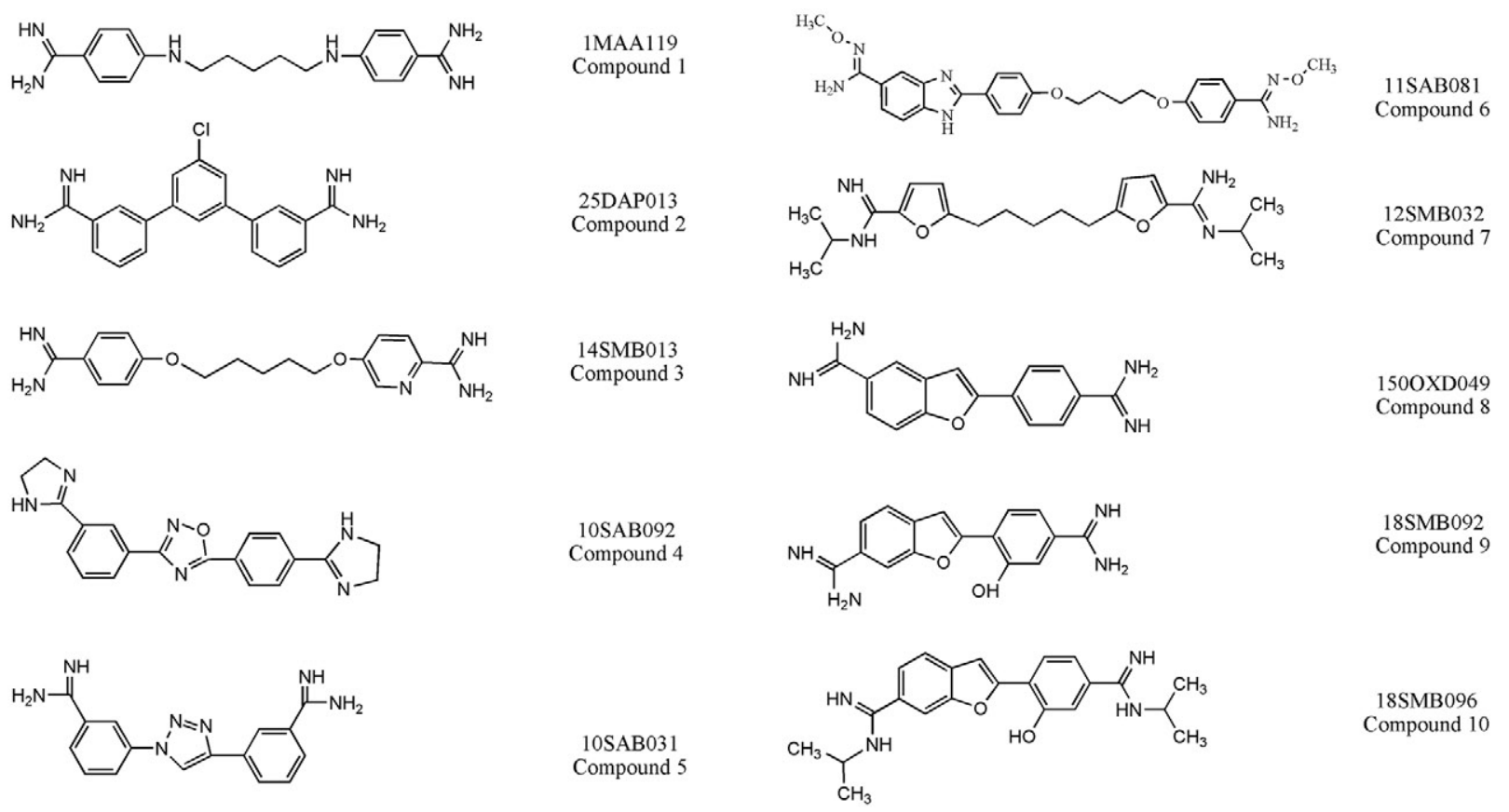

Fig. 1: chemical structure of the compounds.

Cell cultures - Primary cultures of embryonic cardiomyocytes (CM) were obtained from Swiss mice as previously described (Meirelles et al. 1986). After purification, the CM were seeded at a density of $5 \times$ $10^{4}$ cells/well in 96-well microplates containing gelatincoated cover slips and sustained in Dulbecco's modified medium (DMEM) supplemented with 10\% horse serum, $5 \%$ foetal bovine serum (FCS), $2.5 \mathrm{mM} \mathrm{CaCl}_{2}$, $1 \mathrm{mM} \mathrm{L}$-glutamine and $2 \%$ chicken embryo extract as described previously (Meirelles et al. 1986). The cultures were maintained at $37^{\circ} \mathrm{C}$ in an atmosphere of $5 \%$ $\mathrm{CO}_{2}$ and air and the assays were performed at least three times with duplicate samples. All procedures were carried out in accordance with the guidelines established by the Fiocruz Committee of Ethical for the Use of Animals (CEUA 0099/01).

Parasites - Bloodstream trypomastigotes from the Y strain of T. cruzi were harvested by heart puncture from infected Swiss mice at the parasitaemia peak (Meirelles et al. 1982).

Trypanocidal assays - For the analysis of the effect of the compounds on the bloodstream trypomastigotes, $5 \times 10^{6}$ parasites $/ \mathrm{mL}$ were incubated for $24 \mathrm{~h}$ at $37^{\circ} \mathrm{C}$ in RPMI 1640 medium supplemented with $10 \%$ FCS, in the presence or absence of serial dilutions of the compounds (0.1-32 $\mu \mathrm{M})$. Alternatively, the treatment was performed using trypomastigotes cultured in freshly isolated mouse blood at $4^{\circ} \mathrm{C}$ for $24 \mathrm{~h}$ with the drugs at concentrations up to $32 \mu \mathrm{M}$. The parasite death rates were determined through direct analysis by light microscopy using a Neubauer chamber and the $\mathrm{IC}_{50}$ values (the compound concentration that reduces the number of parasites by $50 \%$ ) were calculated (Silva et al. 2007b).
Infection assays and effect on intracellular parasites For the analysis of the effects of the drugs on intracellular parasites, after $24 \mathrm{~h}$ of parasite-host cell interaction (ratio of 10:1), the infected cultures were washed to remove free parasites and then maintained at $37^{\circ} \mathrm{C}$ in an atmosphere of $5 \% \mathrm{CO}_{2}$ and air in the presence of the compounds $(0.1$ to $32 \mu \mathrm{M})$. The medium plus drug was replaced every 24 h. After $72 \mathrm{~h}$ of treatment, which corresponded to $96 \mathrm{~h}$ of infection, the supernatant was recovered, the number of released parasites was determined by direct quantification using light microscopy and a Neubauer chamber and the $\mathrm{IC}_{50}$ values were calculated.

Cytotoxicity assays - To measure the toxic effects on the host cell, uninfected CM were incubated with the compounds (up to $96 \mu \mathrm{M}$ in DMEM) for $24 \mathrm{~h}$ and $72 \mathrm{~h}$ at $37^{\circ} \mathrm{C}$ and then the cell morphology and viability were evaluated by light microscopy and the method of transcriptional and translational (MTT) colorimetric assay, respectively (Mosmann 1983). The absorbance was measured at $490 \mathrm{~nm}$ in a spectrophotometer (VERSAmax tunable, Molecular devices, USA) and was directly proportional to the cell viability, from which the $\mathrm{LC}_{50}$ values (the compound concentration that reduces cellular viability by $50 \%$ ) were calculated.

Fluorescence microscopic analysis and fluorescent intensity determination - The bloodstream forms were treated for $30 \mathrm{~min}$ at $37^{\circ} \mathrm{C}$ with $10 \mu \mathrm{g} / \mathrm{mL}$ of each compound, fixed with $4 \%$ paraformaldehyde and mounted with 2.5\% 1.4-diazabicyclo-(2.2.2)octane (DABCO) on a slide covered with poly-L-lysine (Sigma Aldrich Corp). The fluorescence was analysed using a Zeiss photomicroscope equipped with epifluorescence (Zeiss Inc, Thornwood, NY). The fluorescence intensity of the 

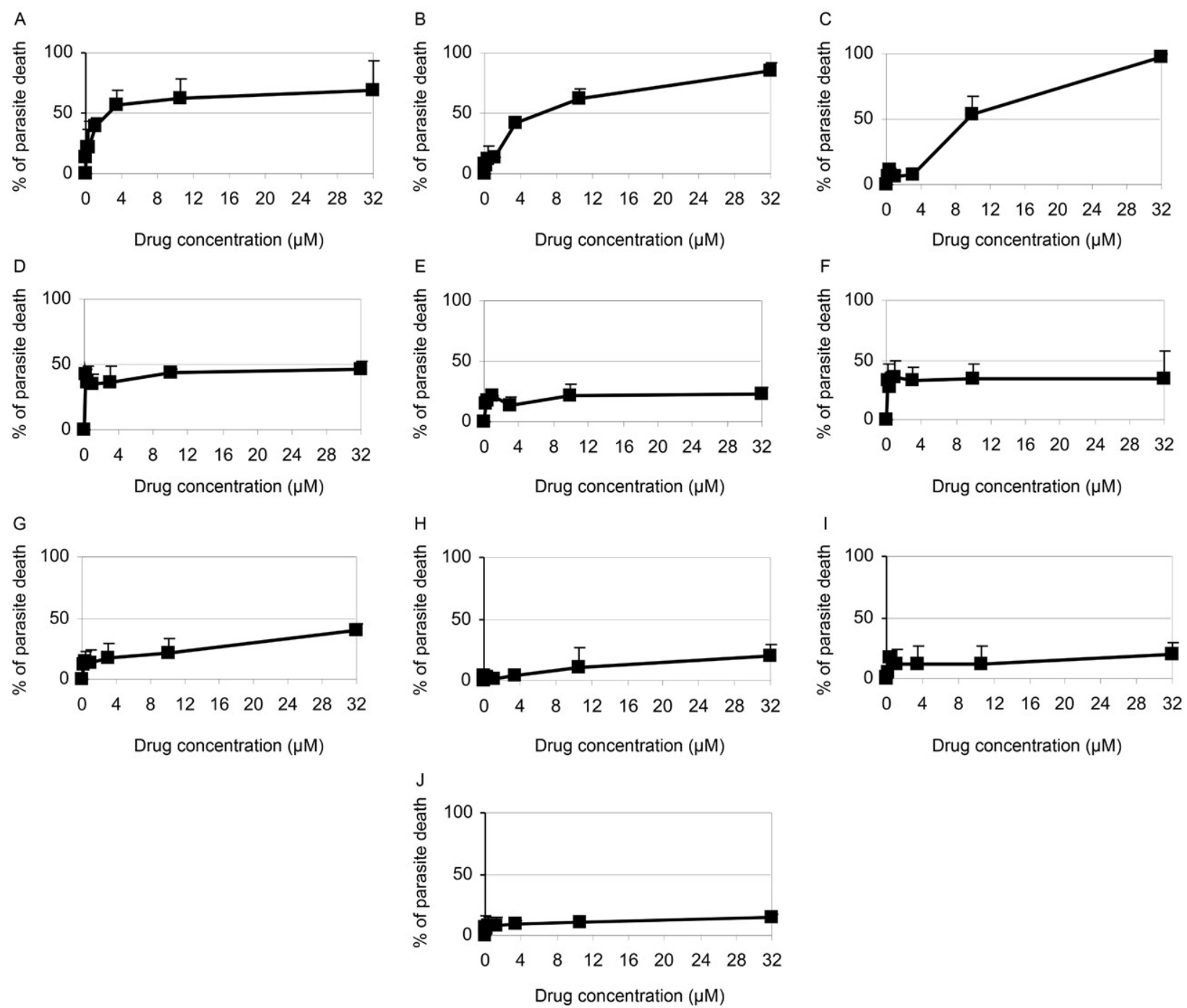

Fig. 2: effect of (A) Compound 1, (B) Compound 2, (C) Compound 3, (D) Compound 4, (E) Compound 5, (F) Compound 6, (G) Compound 7, (H) Compound 8, (I) Compound 9 and (J) Compound 10 on bloodstream trypomastigotes of Trypanosoma cruzi (Y strain) in vitro. The activity was evaluated during the treatment at $37^{\circ} \mathrm{C}$ with the drugs diluted in culture medium. The percentage of dead parasites was measured after $24 \mathrm{~h}$ of treatment.

treated parasites was determined using the program Image J 1.41 (NHI, Bethesda, Maryland) as the sum of the fluorescent pixel values in the selected regions (nucleus DNA - nDNA; kinetoplast DNA - kDNA). The results were expressed as the means and standard deviations of the kDNA/nDNA ratios, which reflect the partition of the kDNA and nDNA fluorescence measurements of at least 50 individual parasites.

\section{RESULTS}

We first evaluated the direct effect of the aromatic dicationic compounds on trypomastigotes, which represent the main infective stage of T. cruzi (Fig. 2). The most active compounds, Compounds 1, 2 and 3, displayed dosedependent effects, with $\mathrm{IC}_{50}$ values of $2.3,6.1$ and $9.3 \mu \mathrm{M}$, respectively (Table I) and about 70,85 and $97 \%$ parasite death at a dose of $32 \mu \mathrm{M}$ (Fig. 2A-C). The other seven

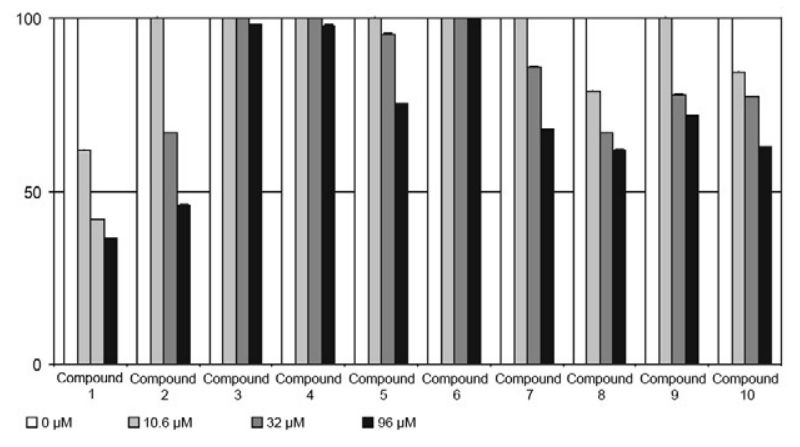

Fig. 3: effect of aromatic dicationic compounds in vitro upon primary cultures of cardiac cells assessed by method of transcriptional and translational (MTT) colorimetric assay. Cardiomyocytes were treated with 10.6, 32 and $96 \mu \mathrm{M}$ of each compound for $72 \mathrm{~h}$. Data are expressed as mean $\pm \mathrm{SD}$ of the percentage of survival in drug-treated cells compared to untreated controls. 
TABLE I

$\mathrm{IC}_{50}$ and selectivity index (SI) values for the effect of aromatic compounds on Ttypanosoma cruzi

\begin{tabular}{|c|c|c|c|c|c|}
\hline & \multicolumn{3}{|c|}{$\begin{array}{c}\text { Trypomastigotes }^{a} \\
24 \mathrm{~h}\end{array}$} & \multicolumn{2}{|c|}{$\begin{array}{l}\text { Intracellular parasites }{ }^{b} \\
\qquad 72 \mathrm{~h}\end{array}$} \\
\hline & $\begin{array}{c}\mathrm{IC}_{50}(\mu \mathrm{M}) \\
4^{\circ} \mathrm{C}\end{array}$ & $\begin{array}{c}\mathrm{IC}_{50}(\mu \mathrm{M}) \\
37^{\circ} \mathrm{C}\end{array}$ & SI & $\mathrm{IC}_{50}(\mu \mathrm{M})$ & SI \\
\hline Compound 1 & $>32.0$ & 2.3 & $>40.0$ & 10.6 & 2.3 \\
\hline Compound 2 & $>32.0$ & 6.1 & $>15.0$ & $>32.0$ & 2.7 \\
\hline Compound 3 & $>32.0$ & 9.3 & $>10.0$ & 0.6 & $>160.0$ \\
\hline Compound 4 & $>32.0$ & $>32.0$ & 3.0 & 0.1 & $>960.0$ \\
\hline Compound 5 & $>32.0$ & $>32.0$ & 3.0 & 0.3 & $>331.0$ \\
\hline Compound 6 & $>32.0$ & $>32.0$ & 3.0 & 2.3 & $>43.0$ \\
\hline Compound 7 & $>32.0$ & $>32.0$ & 3.0 & 0.8 & $>126.0$ \\
\hline Compound 8 & $>32.0$ & $>32.0$ & 3.0 & 20.0 & $>4.7$ \\
\hline Compound 9 & $>32.0$ & $>32.0$ & 3.0 & 20.0 & $>4.9$ \\
\hline Compound 10 & $>32.0$ & $>32.0$ & 3.0 & $>32.0$ & 3.0 \\
\hline
\end{tabular}

SI corresponds to the ratio $\mathrm{LC}_{50} / \mathrm{IC}_{50}$. $a$ : direct effect of the compounds on trypomastiotes performed after $24 \mathrm{~h}$ of incubation at $4^{\circ} \mathrm{C}$ in whole blood or at $37^{\circ} \mathrm{C}$, in RPMI medium; $b$ : effect on intracellular parasites measured by trypomastigotes release into the supernatant culture medium ( $96 \mathrm{~h}$ of infection) performed after $72 \mathrm{~h}$ of treatment at $37^{\circ} \mathrm{C}$.

compounds displayed only modest activities, with $\mathrm{IC}_{50}$ values higher that $32 \mu \mathrm{M}$ (Fig. 2D-J, Table I). However, when the bloodstream forms were exposed to Compounds 1,2 and 3 in the presence of freshly isolated mouse blood, which tested the possible application of these compounds for the prophylaxis of banked blood, we observed a substantial decrease in the trypanocidal activities, with $\mathrm{IC}_{50}$ values higher than $32 \mu \mathrm{M}$ (Table I).

Next, to evaluate the toxicity on mammalian host cells, uninfected cardiac cultures were incubated for 24 and $72 \mathrm{~h}$ with different doses of the compounds and then cellular viability was evaluated by both light microscopy and the MTT colorimetric assay. The compounds did not induce loss of cellular viability after incubation for $24 \mathrm{~h}$ with doses up to $96 \mu \mathrm{M}$ (data not shown); however, most of the aromatic dicationic compounds displayed low toxicity after $72 \mathrm{~h}$ of incubation and Compounds 1 and 2 exhibited moderate toxicity, with $\mathrm{LC}_{50}$ values of 25 and $85 \mu \mathrm{M}$, respectively (Fig. 3).

Next, the anti-parasitic activity of the compounds against the intracellular forms of $T$. cruzi was assessed through the direct quantification of the number of parasites released in the supernatant of infected CM after 96 $\mathrm{h}$ of parasite interaction. Incubation for $72 \mathrm{~h}$ with Compounds 7, 4, 3, 6 and 5 resulted in dose-dependent effects that lead to considerable reductions in the number of parasites released into the supernatant, with micromolar and sub-micromolar $\mathrm{IC}_{50}$ values (Fig. $4 \mathrm{C}-\mathrm{G}$, Table I). On the other hand, Compounds 1, 8 and 9 exerted moderated activity while Compounds 2 and 10 were not active and had $\mathrm{IC}_{50}$ values higher that $32 \mu \mathrm{M}$ (Fig. 4A-B,
H-J, Table I). With the exceptions of Compounds 1 and 2 , the other compounds displayed equal or better activity on intracellular parasites compared to the bloodstream parasites (Table I).

Based on the $\mathrm{IC}_{50}$ and $\mathrm{LC}_{50}$ values, the selectivity index (SI) of each compound was determined. This parameter reflects the quantity of compound that is active against the pathogen but is not toxic towards the host cell. For the bloodstream trypomastigotes, only one dicationic compound (Compound 1) showed a high SI value ( $>40)$, but for the intracellular parasites, five out of 10 compounds displayed considerable selectivity: Compounds 7, 4, 3, 6 and 5 with SI ranging between $>43$ and $>960$. These five aromatic compounds also displayed higher anti-proliferative effects on the intracellular parasites.

Within the treated bloodstream parasites, all of the fluorescent compounds were localised in DNA-enriched structures, i.e., the kinetoplast and nucleus (Fig. 5). However, although there was consistently higher labelling within the kDNA compared to the nuclei (Fig. 5), the $\mathrm{kDNA} / \mathrm{nDNA}$ ratios showed that the higher accumulation in the kDNA (ratios $\geq 1.28$ ) did not correlate with compound efficacy: Compound 7, one of the less active compounds, showed the highest accumulation in the kinetoplast, with a $1.77 \mathrm{kDNA} / \mathrm{nDNA}$ ratio (Table II).

\section{DISCUSSION}

Diamidines and related dications are considered to be potential anti-parasitic agents due to their known activities against several pathogens (Soeiro et al. 2005). However, as they possess critical limitations regarding 
A

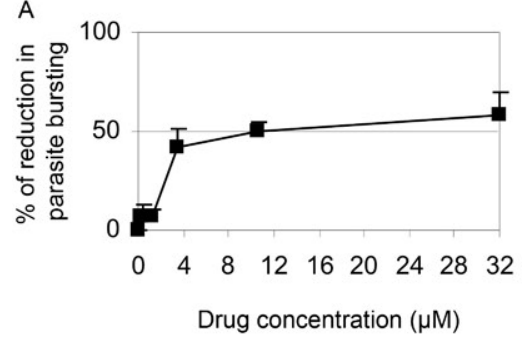

D

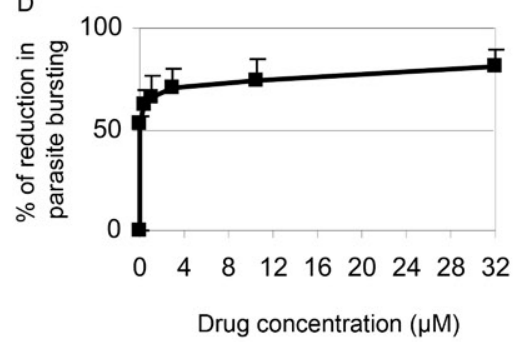

G

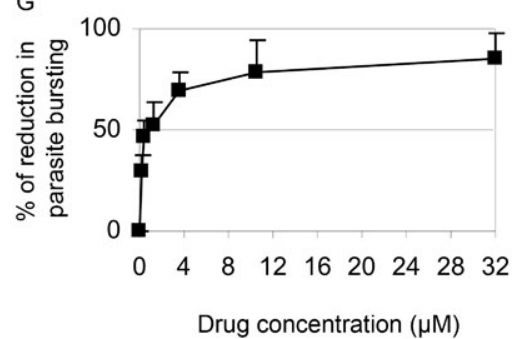

B

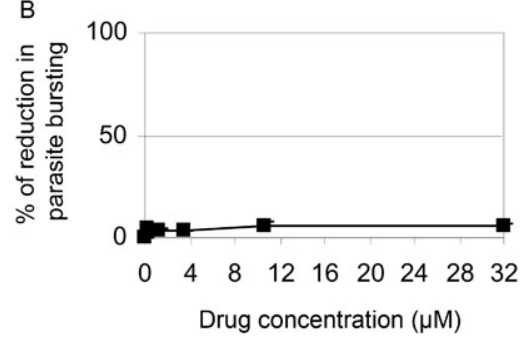

E

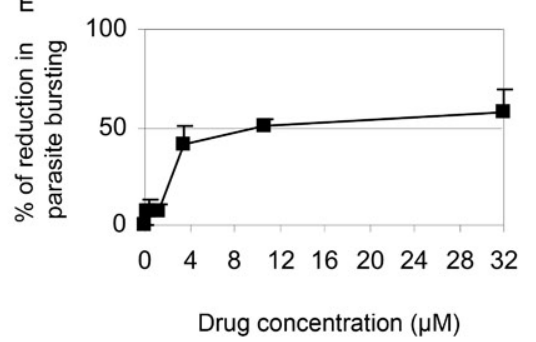

$\mathrm{H}$

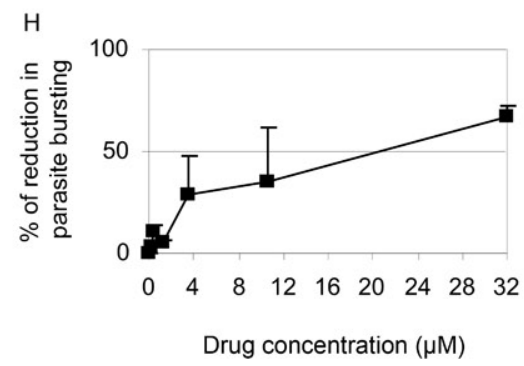

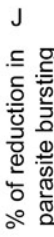

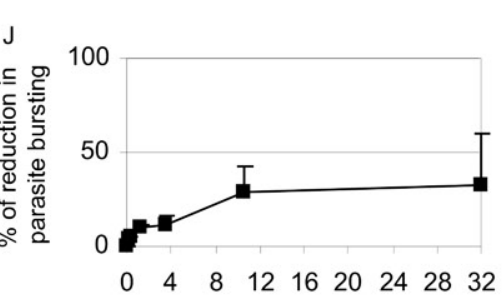

C

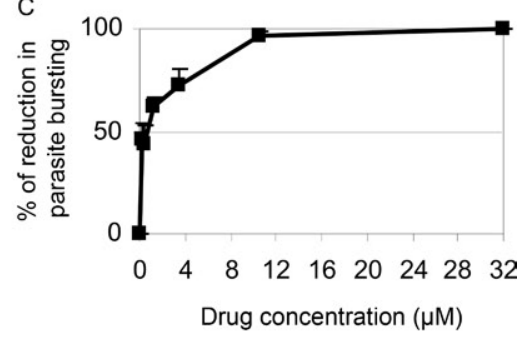

F

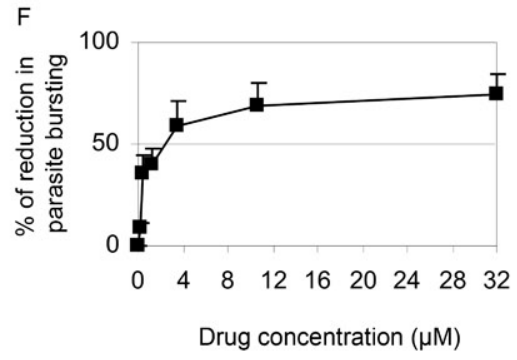

I

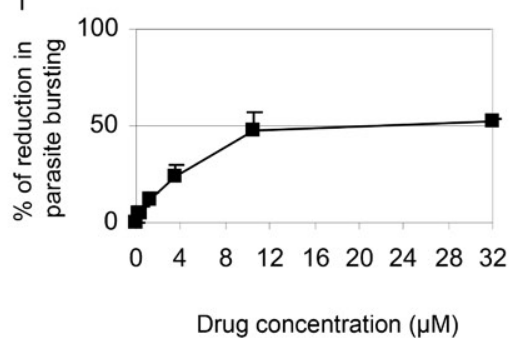

Fig. 4: activity of (A) Compound 1, (B) Compound 2, (C) Compound 3, (D) Compound 4, (E) Compound 5, (F) Compound 6, (G) Compound 7, (H) Compound 8, (I) Compound 9 and (J) Compound 10 upon intracellular parasites lodge in Trypanosoma cruzi-infected cardiac cells. The activity of compounds after $72 \mathrm{~h}$ of drug incubation is shown by the percentage of reduction in the number of released parasites into the supernatant of the infected cultures.

their poor oral bioavailability and considerable toxicity, new dicationic analogs have been synthesised to address this situation.

Our assays evaluated the effect of 10 aromatic dicationic compounds on trypomastigotes under different experimental conditions to explore their potential uses as chemotherapeutics (assays conducted at $37^{\circ} \mathrm{C}$ ) and $/$ or prophylactic compounds for banked blood (assays using whole blood at $4^{\circ} \mathrm{C}$ ). Our data showed that although three compounds, Compounds 1, 2 and 3, induced high levels of parasite lysis and dose-dependent effects with low micromolar $\mathrm{IC}_{50}$ values when assayed at $37^{\circ} \mathrm{C}$, all of them showed decreased activity in the presence of blood, possibly due to their association with and/or inactivation by serum components as reported previously (SantaRita et al. 2004, 2006, Silva et al. 2007a). Therefore, the decreased activity at $4^{\circ} \mathrm{C}$ in the presence of blood constituents demonstrated that the studied compounds are ineffective for the sterilisation of ex vivo blood batches to control Chagas disease.

In agreement with our previous studies showing that reversed amidines, also named arylimidamides, exhibited low toxicity to mammalian cells in vitro (Silva et al. 2007a), our present data showed that, except for Compounds 1 and 2, only high drug concentrations $(>96$ $\mu \mathrm{M})$ induced alterations in host cell viability.

We also found that five out of 10 Compounds (Compounds 7, 4, 3, 6 and 5) exerted considerable activity against the intracellular forms of $T$. cruzi at low micromolar and sub-micromolar doses and with high SI values (ranging between $>43$ and $>960$ ). This difference in activity on the intracellular forms compared to the 


\section{TABLE II}

Mean and standard deviation values of fluorescence intensity ratios among kinetoplast and nuclei of bloodstream trypomastigotes treated for $30 \mathrm{~min}$ with $10 \mu \mathrm{g} / \mathrm{mL}$ of each compound

\begin{tabular}{lc}
\hline & Kinetoplast/nucleus \\
\hline Compound 1 & $1.60 \pm 0.44$ \\
Compound 2 & $1.61 \pm 0.36$ \\
Compound 3 & $1.58 \pm 0.38$ \\
Compound 4 & $1.50 \pm 0.32$ \\
Compound 5 & $1.61 \pm 0.30$ \\
Compound 6 & $1.48 \pm 0.31$ \\
Compound 7 & $1.77 \pm 0.38$ \\
Compound 8 & $1.28 \pm 0.33$ \\
Compound 9 & $1.53 \pm 0.30$ \\
Compound 10 & $1.39 \pm 0.35$ \\
\hline
\end{tabular}

bloodstream forms requires further analysis but could represent differences in drug uptake by these different parasite stages and/or different mechanisms of action upon non-dividing trypomastigotes and the highly multiplicative intracellular stages of the parasite.

Aromatic dicationic compounds, such as pentamidine, bind non-covalently and in a non-intercalative manner to the minor-groove of the DNA; however, their mechanism of action has not been fully elucidated and it has been proposed that they may possess multiple modes of action (Wilson et al. 2005). One of the long-hypothesised mechanisms of action of diamidines is related to their ability to bind to AT-rich regions of the DNA minor groove, but other mechanisms have also been proposed, such as inhibition of tyrosyl-DNA phosphodiesterase, topoisomerases, protein kinase A, proteases and polymerases (Tidwell \& Boykin 2003, Soeiro et al. 2008, Soeiro \& De Castro 2009).

According to our present results, we could not find any correlation between the localisation and higher accumulation of these dicationic fluorescent compounds within the $T$. cruzi kDNA and their trypanocidal activity, which we also found in another recent study of other dicationic compounds (Daliry et al. 2009). In fact, previous reports on African trypanosomes also could not correlate either intracellular accumulation or sub cellular localisation and distribution of aza analogs and diphenyl furans with their in vitro activities (Mathis et al. 2007).

Our present paper describes the potential effect of the aromatic dicationic compounds on T. cruzi, which supports further screening of new analogs that could be used alone or in combination with other drugs for the treatment of Chagas disease.

\section{REFERENCES}

Daliry A, Silva PB, Silva CF, Meuser MB, de Castro SL, Tidwell $\mathrm{RR}$, Soeiro MNC 2009. In vitro analyses of the effect of aromatic diamidines upon Trypanosoma cruzi. J Antimicrob Chemother 64: 747-750.

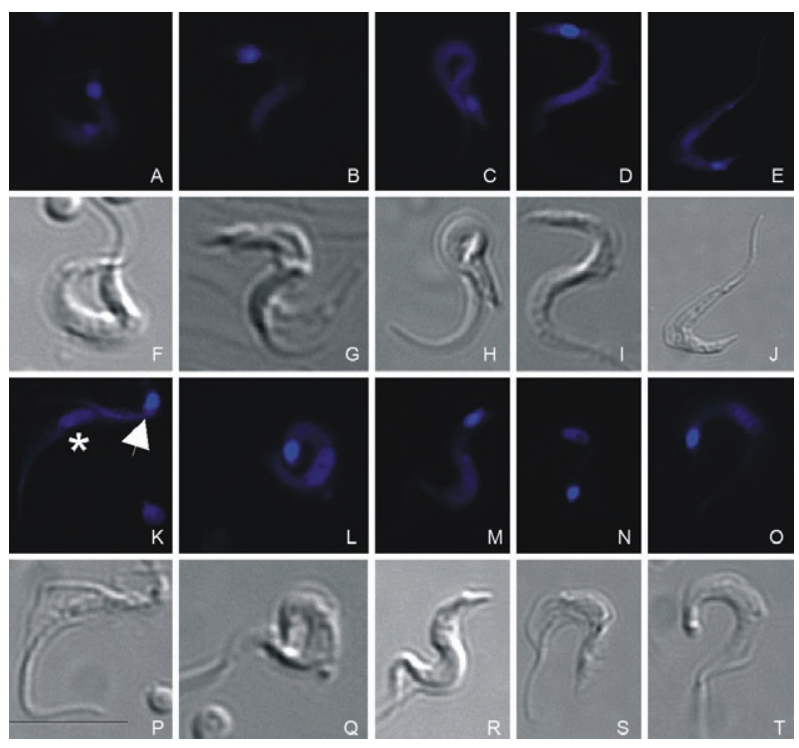

Fig. 5: fluorescent (A-E, K-O) and differential interference contrast (F-J, P-T) analysis showing intracellular localization of the aromatic dicationic compounds within bloodstream trypomastigotes of Trypanosoma cruzi after incubation for $30 \mathrm{~min}$ at the concentration of 10 $\mu \mathrm{g} / \mathrm{mL}$ : Compound 1 (A, F), Compound 2 (B, G), Compound 3 (C, H), Compound 4 (D, I), Compound 5 (E, J), Compound 6 (K, P), Compound $7(\mathrm{~L}, \mathrm{Q})$, Compound $8(\mathrm{M}, \mathrm{R})$, Compound $9(\mathrm{~N}, \mathrm{~S})$ and Compound $10(\mathrm{O}, \mathrm{T})$. Note that compound accumulation was higher in the kinetoplast (white arrow) than in the nucleus (asterisk). Bar $=2 \mu \mathrm{m}$.

da Silva CF, Batista MM, Batista D da G, de Souza EM, da Silva PB, de Oliveira GM, Meuser AS, Shareef AR, Boykin DW, Soeiro M de N 2008. Trypanocidal activity of a diarylthiophene diamidine against Trypanosoma cruzi: in vitro and in vivo studies. Antimicrob Agents Chemother 52: 3307-3314.

De Souza EM, Lansiaux A, Bailly C, Wilson WD, Hu Q, Boykin DW, Batista MM, Araújo-Jorge TC, Soeiro MN 2004. Phenyl substitution of furamidine markedly potentiates its antiparasitic activity against Trypanosoma cruzi and Leishmania amazonensis. Biochem Pharmacol 68: 593-600.

De Souza EM, Menna-Barreto R, Araújo-Jorge TC, Kumar A, Hu Q, Boykin DW, Soeiro, MNC 2006a. Antiparasitic activity of aromatic diamidines is related to apoptosis-like death in Trypanosoma cruzi. Parasitology 133: 75-79.

De Souza EM, Oliveira GM, Boykin DW, Kumar A, Hu Q, Soeiro MNC 2006b. Trypanocidal activity of the phenyl-substituted analogue of furamidine DB569 against Trypanosoma cruzi infection in vivo. J Antimicrob Chemother 58: 610-614.

Dias JC 2007. Southern Cone Initiative for the elimination of domestic populations of Triatoma infestans and the interruption of transfusion Chagas disease: historical aspects, present situation and perspectives. Mem Inst Oswaldo Cruz 102: 11-18.

Jannin J, Villa L 2007. An overview of Chagas disease treatment. Mem Inst Oswaldo Cruz 102: 95-97.

Mathis AM, Bridges AS, Ismail MA, Kumar A, Francesconi I, Anbazhagan M, Hu Q, Tanious FA, Wenzler T, Saulter J, Wilson WD, Brun R, Boykin DW, Tidwell RR, Hall JE 2007. Diphenyl furans and aza analogs: effects of structural modification on in vitro activity, DNA binding and accumulation and distribution in trypanosomes. Antimicrob Agents Chemother 51: 2801-2810. 
Meirelles MN, de Araújo Jorge TC, de Souza W 1982. Interaction of Trypanosoma cruzi with macrophages in vitro: dissociation of the attachment and internalization phases by low temperature and cytochalasin B. Z Parasitenkd 68: 7-14.

Meirelles MN, de Araujo-Jorge TC, Miranda CF, de Souza W, Barbosa HS 1986. Interaction of Trypanosoma cruzi with heart muscle cells: ultrastructural and cytochemical analysis of endocytic vacuole formation and effect upon myogenesis in vitro. Eur $J$ Cell Biol 41: 198-206.

Mosmann T 1983. Rapid colorimetric assay for cellular growth and survival: application to proliferation and cytotoxicity assays. J Immunol Methods 65: 55-63.

Rocha MO, Teixeira MM, Ribeiro AL 2007. An update on the management of Chagas cardiomyopathy. Expert Rev Anti Infect Ther 5: $727-743$.

Rodriques Coura RJ, de Castro SL 2002. A critical review on Chagas disease chemotherapy. Mem Inst Oswaldo Cruz 97: 3-24.

Santa-Rita RM, Barbosa HS, de Castro SL 2006. Ultrastructural analysis of edelfosine-treated trypomastigotes and amastigotes of Trypanosoma cruzi. Parasitol Res 100: 187-190.

Santa-Rita RM, Santos Barbosa H, Meirelles MN, de Castro SL 2004. Effect of the alkyl-lysophospholipids on the proliferation and differentiation of Trypanosoma cruzi. Acta Trop 75: 219-228.

Silva CF, Batista MM, Mota RA, de Souza EM, Stephens CE, Som P, Boykin DW, Soeiro MdeN 2007a. Activity of "reversed" diamidines against Trypanosoma cruzi in vitro. Biochem Pharmacol 73: 1939-1946.

Silva CF, Meuser MB, De Souza EM, Meirelles MN, Stephens CE, Som P, Boykin DW, Soeiro MN 2007b. Cellular effects of reversed amidines on Trypanosoma cruzi. Antimicrob Agents Chemother 51: 3803-3809. Soeiro MNC, De Castro SL, De Souza EM, Batista
DGJ, Silva CF, Boykin DW 2008. Diamidine activity against trypanosomes: the state of the art. Curr Mol Pharmacol 1: 151-161.

Soeiro MN, Dantas AP, Daliry A, Silva CF, Batista DG, de Souza EM, Oliveira GM, Salomão K, Batista MM, Pacheco MG, Silva PB, Santa-Rita RM, Barreto RF, Boykin DW, Castro SL 2009. Experimental chemotherapy for Chagas disease: 15 years of research contributions from in vivo and in vitro studies. Mem Inst Oswaldo Cruz 104: 301-310.

Soeiro MN, De Castro SL 2009. Trypanosoma cruzi targets for new chemotherapeutic approaches. Expert Opin Ther Targets 13: 105-121.

Soeiro MN, De Souza EM, Stephens CE, Boykin DW 2005. Aromatic diamidines as antiparasitic agents. Expert Opin Investig Drugs 14: 957-972.

Stewart M, Krishna S, Burchmore RS, Brun R, de Koning HP, Boykin DW, Tidwell RR, Hall JE, Barrett MP 2005. Detection of arsenical drug resistance in Trypanosoma brucei with a simple fluorescence test. Lancet 366: 486-487.

Tidwell RR, Boykin DW 2003. Minor groove binders as antimicrobial agents. In M Demeunynck, C Bailly, WD Wilson (eds.), Small molecule DNA and RNA binder: synthesis to nucleic acid complexes, Wiley-VCH, New York, p. 416-460.

Urbina JA 2002. Chemotherapy of Chagas disease. Curr Pharm Des 8: 287-295.

Werbovetz K 2006. Diamidines as antitrypanosomal, antileishmanial and antimalarial agents. Curr Opin Investig Drugs 7: 147-157.

Wilson WD, Nguyen B, Tanious FA, Mathis A, Hall JE, Stephens CE, Boykin DW 2005. Dications that target the DNA minor groove: compound design and preparation, DNA interactions, cellular distribution and biological activity. Curr Med Chem Anticancer Agents 5: 389-408.

Wilson WD, Tanious FA, Mathis A, Tevis D, Hall JE, Boykin DW 2008. Antiparasitic compounds that target DNA. Biochimie 90: 999-1014. 